Continuum quantum systems as limits of discrete quantum systems. IV. Affine canonical transforms

Laurence Barker

Citation: Journal of Mathematical Physics 44, 1535 (2003); doi: 10.1063/1.1557331

View online: http://dx.doi.org/10.1063/1.1557331

View Table of Contents: http://aip.scitation.org/toc/jmp/44/4

Published by the American Institute of Physics

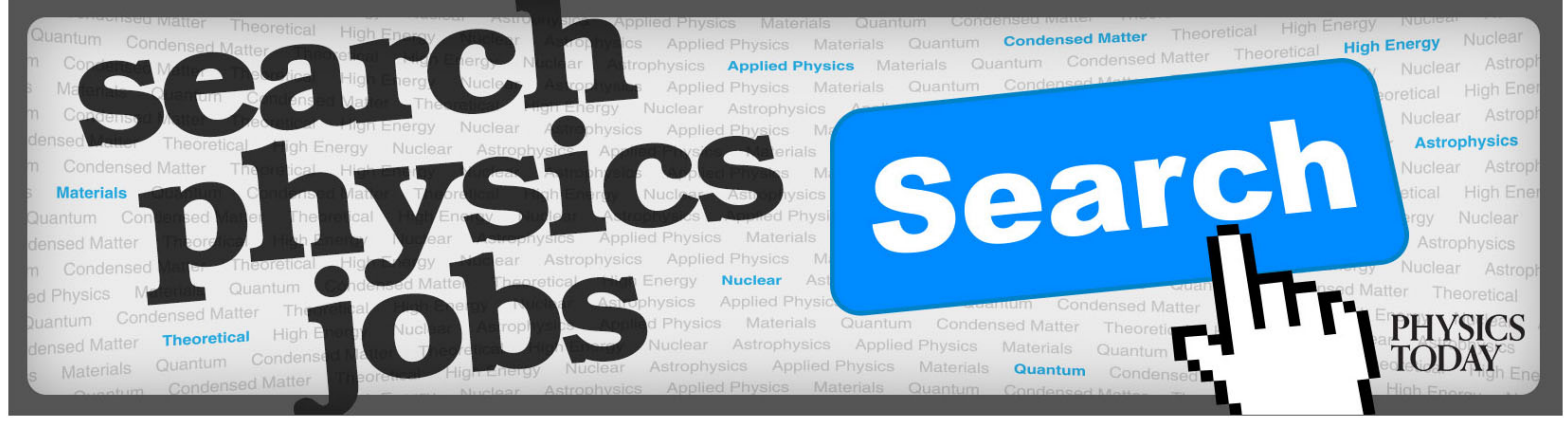




\title{
Continuum quantum systems as limits of discrete quantum systems. IV. Affine canonical transforms
}

\author{
Laurence Barker ${ }^{a)}$ \\ Department of Mathematics, Bilkent University, 06533 Bilkent, Ankara, Turkey
}

(Received 4 June 2002; accepted 30 December 2002)

\begin{abstract}
Affine canonical transforms, complex-order Fourier transforms, and their associated coherent states appear in two scenarios: finite-discrete and continuum. We examine the relationship between the two scenarios, making systematic use of inductive limits, which were developed in the preceding articles in this series. (C) 2003 American Institute of Physics. [DOI: 10.1063/1.1557331]
\end{abstract}

\section{INTRODUCTION}

Inductive limits provide a clear and precise means whereby objects associated with a continuum system can be realized as limits of objects associated with a sequence of discrete systems. Three preceding papers ${ }^{1-3}$ discuss inductive limits of vectors and operators. Another work ${ }^{4}$ concerns inductive limits of representations. In the present article, we illustrate the approach by applying it to a continuum scenario and a discrete scenario that lie in the core of quantum physics.

Our main results are as follows. Theorem 6.1 realizes Glauber coherent states as inductive limits of spin coherent states. A practical version of the result goes back to Radcliffe ${ }^{5}$ and Arecchi et al. ${ }^{6}$ Theorem 5.3 realizes the group of continuum motion canonical transforms as an inductive limit of the group of discrete motion canonical transforms. A practical version was initiated in Ref. 6 and considerably developed by Atakishiyev et al. ${ }^{7.8}$ Theorem 5.1 and Corollary 5.2 realize single-parameter groups of continuum affine canonical transforms as inductive limits of singleparameter groups of discrete affine canonical transforms. Practical versions can be found in Dobrev et al. ${ }^{9}$ Theorem 6.2 realizes continuum complex-order Fourier transforms as inductive limits of discrete complex-order Fourier transforms. From a practical point of view, that can be seen as a mild generalization of the fractional Fourier transforms in Ref. 10. In Ref. 4, Corollary 5.2 and Theorem 5.3 are expressed explicitly as inductive limits of representations but, in the present article, they are expressed simply as inductive limits of operators.

In using the adjective "practical," rather than "heuristic," we have erred towards understatement rather than overstatement. There is a vast body of literature on discrete to continuum correspondences that seem to be potential applications of inductive limits; see Sec. VII for a sample of further citations. Sometimes, in those works, the practical versions of the results have involved expressions of the form $\mathcal{O}=\lim _{n} \mathcal{O}_{n}$ or $\mathcal{O}_{n} \rightarrow \mathcal{O}$ that do not conform to any evident definition of limit. Sometimes, comparatively weak results have been stated and proved, yet with an apparently suggested meaning that goes beyond the literal interpretation; for instance, parallel discussion of continuum and discrete scenarios, the latter implicitly understood to be an approximation to the former. Actually, our use of inductive limits does have a practical intention, as we shall explain in Sec. VII.

Let us indicate the nature of the general kind of problem that concerns us. The limit equations in question are of the form $\mathcal{O}=\lim _{n} \mathcal{O}_{n}$, where $\mathcal{O}$ is an object (say, a vector, an operator or a representation) associated with Hilbert space $\mathcal{L}$, and each $\mathcal{O}_{n}$ is an object associated with a Hilbert space $\mathcal{L}_{n}$. In this article, $\mathcal{L}=L^{2}(\mathbb{R})$ and $\mathcal{L}_{n}$ is of finite dimension $n$. The problem is to select appropriate definitions so as to make such limit equations potentially provable or refutable; or, at least, true or false. One approach is to embed the spaces $\mathcal{L}_{n}$ in the space $\mathcal{L}$, and to replace

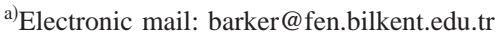


differential equations with corresponding difference equations. In general form, this is, of course, a numerical approximation technique that has been in widespread use ever since the emergence of statistical analysis in the 18th century. We must be very selective with our citations, since otherwise there would be no end to them. The convergence of eigenvectors examined in Ref. 11 may be applicable to the operators we consider below; this is significant, because convergence of spectral measures may be an interesting avenue for research into discrete to continuum limits of representations (see Ref. 3, Sec. V). In Ref. 12, groups acting on $\mathcal{L}_{n}$ are embedded in groups acting on $\mathcal{L}$, and the discrete to continuum correspondence is characterized in terms of module induction. Another approach, proposed by Parthasarathy ${ }^{13,14}$ and Lindsay-Parthasarathy, ${ }^{15}$ is to collect all the spaces $\mathcal{L}_{n}$ together in a Fock space where limits can be examined without mentioning the space $\mathcal{L}$. Arguably, our approach is the most flexible of the three, since the definitions of inductive limits of vectors and operators do not require any constraints on the Hilbert spaces $\mathcal{L}$ and $\mathcal{L}_{n}$ (except for separability). However, it seems very probable that the particular limit equations in the present article can also be realized through the other two approaches.

Although some of the material below is in the nature of a review, this is a side-effect of a need to reformulate known results before presenting our own. We must also point out that although some of our limit formulas are unitary versions of accepted heuristic limits of Hermitian operators, the assertions that the formulas now express are new, since the kinds of limit involved had not previously been supplied with definitions.

\section{CONTINUUM AFFINE CANONICAL TRANSFORMS}

We shall introduce a six-dimensional connected real Lie group $\operatorname{HSA}=\operatorname{HSA}(2, \mathrm{R})$ and an action of HSA as unitary operators on the continuum state space $L^{2}(\mathbb{R})$. As we shall see in the next section, HSA is a central extension of the special affine group SA on the plane; SA is also the Schorödinger group with one space dimension and one time dimension. The group HSA, and its representation on $L^{2}(\mathbb{R})$, are discussed by Dobrev et al. ${ }^{9}$ and Neiderer, ${ }^{16}$ for some other sources-oriented more towards the phase space picture-see Sec. III. Our main target, in this section, is to obtain explicit matrix representations for some generators of the Lie algebra of HSA. We shall also examine a subgroup HM of HSA. The group HM is a central extension of the Euclidian motion group.

The real Lie algebra hsa $=\mathrm{hsa}(2, \mathrm{R})$ has a basis $\{i B, i C, i D, i P, i Q, i I\}$. The notation indicates that $B, C, D, P, Q, I$ are elements of the complexification. The commutation relations are defined to be such that $I$ is central, $[Q, P]=i I$ and, in the universal enveloping algebra,

$$
B=\frac{1}{2} P^{2}, \quad C=\frac{1}{2} Q^{2}, \quad D=\frac{1}{2}(P Q+Q P) .
$$

It is not hard to show that the commutation relations involving $B, C, D$ are

$$
\begin{gathered}
{[B, P]=0=[C, Q], \quad[C, P]=i Q=-[D, Q], \quad[D, P]=i P=-[B, Q],} \\
{[B, C]=-i D, \quad[B, D]=-2 i B, \quad[C, D]=2 i C .}
\end{gathered}
$$

For instance,

$$
\begin{aligned}
{[B, C] } & =\frac{1}{4}\left(P^{2} Q^{2}-P Q P Q+P Q P Q-P Q^{2} P+P Q^{2} P-Q P Q P+Q P Q P-Q^{2} P^{2}\right) \\
& =\frac{1}{4}(P[P, Q] Q+P Q[P, Q]+[P, Q] Q P+Q[P, Q] P)=-i D .
\end{aligned}
$$

Let $\hat{B}, \hat{C}, \hat{D}, \hat{P}, \hat{Q}, \hat{I}$ be the Hermitian operators on $L^{2}(\mathbb{R})$ such that $\hat{I}$ is the identity operator and

$$
(\hat{P} \phi)(q)=-i \frac{d}{d q} \phi(q), \quad(\hat{Q} \phi)(q)=q \phi(q),
$$




$$
\hat{B}=\frac{1}{2} \hat{P}^{2}, \quad \hat{C}=\frac{1}{2} \hat{Q}^{2}, \quad \hat{D}=\frac{1}{2}(\hat{P} \hat{Q}+\hat{Q} \hat{P}),
$$

where $\phi$ belongs to the Schwartz space $\mathcal{S}(\mathbb{R})$. The operators $\hat{P}$ and $\hat{Q}$ are sometimes understood to correspond to momentum and position, respectively (or frequency and time, in signal processing, or frequency and position, in optics).

Let $\sigma$ be the anti-Hermitian representation of hsa on $L^{2}(\mathbb{R})$ such that the elements $B, C, D$, $P, Q, I$ act as $\hat{B}, \hat{C}, \hat{D}, \hat{P}, \hat{Q}, \hat{I}$, respectively. We introduce a real Lie group $\operatorname{HSA}=\mathrm{HSA}(2, \mathrm{R})$ and a faithful unitary representation $\rho$ of HSA such that HSA has associated Lie algebra hsa and such that $\rho$ has differential representation $\sigma$. The elements of the group $\rho$ (HSA) are called continuum affine canonical transforms. Of course, there is no essential difference between the abstract Lie group HSA and the group of unitary operators $\rho(\mathrm{HSA})$. Each is isomorphic to the other via the isomorphism $\rho$. Nevertheless, we do sometimes find it useful to distinguish between the two groups. Given real $\beta, \gamma, \delta, \mu, \nu, \kappa$, we write

$$
\begin{gathered}
\hat{H}(\beta, \gamma, \delta, \mu, \nu, \kappa)=\beta \hat{B}+\gamma \hat{C}+\delta \hat{D}+\mu \hat{P}+\nu \hat{Q}+\kappa \hat{I}, \\
\hat{U}(\beta, \gamma, \delta, \mu, \nu, \kappa)=\exp (-i \hat{H}(\beta, \gamma, \delta, \mu, \nu, \kappa)) .
\end{gathered}
$$

The continuum affine canonical transforms are the composites of operators that have the form $\hat{U}(\beta, \ldots, \kappa)$.

Warning: some affine canonical transforms do not have the exponential form $\hat{U}(\beta, \ldots, \kappa)$. We shall not be making use of this negative result, but we mention that it can be proved by considering the subquotient $\operatorname{SL}(2, \mathrm{R})$ of HSA, and using Eq. (19).

As an element of the Lie algebra hsa, we define

$$
N=B+C-I / 2 .
$$

The corresponding Hermitian operator on $L^{2}(\mathbb{R})$ is

$$
\hat{N}=\sigma(N)=\hat{B}+\hat{C}+\hat{I} / 2 .
$$

Let $\mathrm{hm}=\mathrm{hm}(2, \mathrm{R})$ be the subalgebra of hsa with basis $\{i I, i N, i P, i Q\}$ and let $\mathrm{HM}=\mathrm{HM}(2, \mathrm{R})$ be the subgroup of HSA with associated Lie algebra $\mathrm{hm}$. We call HM the group of Heisenberg motions, and we call the elements of the group $\rho(\mathrm{HM})$ the continuum motion canonical transforms. Again, there is no essential difference between the two isomorphic groups $\mathrm{HM}$ and $\rho(\mathrm{HM})$. The commutation relations for HM are given by Eq. (1) together with

$$
[N, I]=0, \quad[N, P]=i Q, \quad[N, Q]=-i P .
$$

The continuum (and discrete) motion canonical transforms will be of particular importance to us, and it is worth introducing some special notation for them. Given $\kappa, \lambda, \mu, \nu \in \mathbb{R}$, we define

$$
\hat{E}(\kappa, \lambda, \mu, \nu)=\exp (-i(\kappa \hat{I}+\lambda \hat{N}+\mu \hat{P}+\nu \hat{Q})) .
$$

By passing to the quotient group $\mathrm{HM} / Z(\mathrm{HM}) \cong \mathrm{EM}$ (see Sec. III), it can easily be shown that the operators having the form $\hat{E}(\kappa, \lambda, \mu, \nu)$ are closed under composition. In other words, the continuum motion canonical transforms are precisely the operators having the form $\hat{E}(\kappa, \lambda, \mu, \nu)$.

We shall give some explicit matrix equations for the infinitesmal generators $\hat{B}, \hat{C}, \hat{D}, \hat{P}, \hat{Q}$, $\hat{I}$ of the continuum affine canonical transforms. For that, we need to specify a complete orthonormal set. Recall that, for $s \in \mathbb{N}$, the $s$-th Hermite polynomial $H_{s}$ and the $s$-th Hermite function $h_{s}$ are the functions $\mathrm{R} \rightarrow \mathrm{C}$ given by 


$$
(-1)^{s} \exp \left(q^{2} / 2\right) \frac{d^{s}}{d q^{s}} \exp \left(-q^{2}\right)=H_{s}(q)=\sqrt{s ! 2^{s} \sqrt{\pi}} \exp \left(q^{2} / 2\right) h_{s}(q) .
$$

Switching to Dirac notation, we write $|s\rangle=h_{s}$. Note that the zeroth Hermite function $|0\rangle=h_{0}$ is the Gaussian function

$$
h_{0}(q)=\pi^{-1 / 4} \exp \left(-q^{2} / 2\right) .
$$

Recall that $\{|s\rangle: s \in \mathbb{N}\}$ is a complete orthonormal set in $L^{2}(\mathbb{R})$. Also recall that the annihilation operator $\hat{A}=(\hat{Q}+i \hat{P}) / \sqrt{2}$ and its Hermitian conjugate, the creation operator $\hat{A}^{\dagger}=(\hat{Q}-i \hat{P}) / \sqrt{2}$, act by

$$
\hat{A}|s\rangle=\sqrt{s}|s-1\rangle, \quad \hat{A}^{\dagger}|s\rangle=\sqrt{s+1}|s+1\rangle .
$$

By direct calculation using Eq. (10), we obtain

$$
\begin{gathered}
\hat{B}|s\rangle=\frac{-1}{4} \sqrt{s(s-1)}|s-2\rangle+\frac{2 s+1}{4}|s\rangle+\frac{-1}{4} \sqrt{(s+1)(s+2)}|s+2\rangle, \\
\hat{C}|s\rangle=\frac{1}{4} \sqrt{s(s-1)}|s-2\rangle+\frac{2 s+1}{4}|s\rangle+\frac{1}{4} \sqrt{(s+1)(s+2)}|s+2\rangle, \\
\hat{D}|s\rangle=\frac{-i}{2} \sqrt{s(s-1)}|s-2\rangle+\frac{i}{2} \sqrt{(s+1)(s+2)}|s+2\rangle, \\
\hat{P}|s\rangle=-i \sqrt{\frac{s}{2}}|s-1\rangle+i \sqrt{\frac{s+1}{2}}|s+1\rangle, \\
\hat{Q}|s\rangle=\sqrt{\frac{s}{2}}|s-1\rangle+\sqrt{\frac{s+1}{2}}|s+1\rangle, \\
\hat{I}|s\rangle=|s\rangle, \\
\hat{N}|s\rangle=s|s\rangle .
\end{gathered}
$$

In Sec. IV, we shall find discrete analogues of these seven matrix equations.

Let us end this section with an example. Recall that the continuum Fourier transform is the unitary operator $\hat{F}$ on $L^{2}(\mathbb{R})$ such that $\hat{F}|s\rangle=i^{s}|s\rangle$. More generally, after Namias, ${ }^{17}$ the continuum fractional Fourier transform of order $t \in \mathbb{R}$ is the unitary operator $\hat{F}^{t}$ on $L^{2}(\mathbb{R})$ such that $\hat{F}^{t}|s\rangle=\exp (2 \pi i s t)|s\rangle$. In other words,

$$
\hat{F}^{t}=\exp (2 \pi i t \hat{N})=e^{-\pi i t} \exp (2 \pi i(\hat{B}+\hat{C})) .
$$

\section{THE CONTINUUM PHASE SPACE PICTURE}

This section has two purposes. One of them is to fulfill the promise, made above, to explain how the groups HSA and HM are central extensions of the groups SA and EM, which act on the real plane. The other purpose is to clarify the relationship between the Hermitian operators and their corresponding unitary operators. In Refs. 6-9 and 12, and many other works, limits are described mainly in terms of Hermitian operators. But inductive limits are defined for unitary operators; they are not defined for unbounded Hermitian operators. So we do need to be able to move freely from Hermitian operators to unitary operators, and in reverse. 
The phase space picture provides much insight into these matters. There is a vast literature on phase space, and much attention has been paid to affine canonical transforms, especially special linear canonical transforms. See, for instance work by Folland, ${ }^{18}$ Hillery et al. ${ }^{19}$ Littlejohn, ${ }^{20}$ Ozorio de Almeida; ${ }^{21}$ we also mention two collections of papers edited by Forbes et al. ${ }^{22}$ (on applications to optics) and Mecklenbräuker-Hlawatsch ${ }^{23}$ (on applications to signal processing). The relevant material, though, is not easy to extract from the literature. Let us give a brief self-contained account of it.

The phase space plane, denoted $\mathcal{P}$, is defined to be a copy of $\mathbb{R}^{2}$. We regard $\mathcal{P}$ as a Euclidean plane equipped with a fixed coordinate system; the vectors are written as coordinate vectors $(p, q)$ where $p$ and $q$ are formal variables.

Recall that the group of special linear transforms of the real plane, denoted $\mathrm{SL}=\mathrm{SL}(2, \mathrm{R})$, has Lie algebra $\mathrm{sl}=\mathrm{sl}(2, \mathrm{R})$ with basis $\{i \bar{B}, i \bar{C}, i \bar{D}\}$ where

$$
\bar{B}=\left(\begin{array}{cc}
0 & -i \\
0 & 0
\end{array}\right), \quad \bar{C}=\left(\begin{array}{cc}
0 & 0 \\
i & 0
\end{array}\right), \quad \bar{D}=\left(\begin{array}{cc}
i & 0 \\
0 & -i
\end{array}\right) .
$$

Thus, SL is generated by the elements having the form

$$
\left(\begin{array}{ll}
a & b \\
c & d
\end{array}\right)=\exp (-i(\beta \bar{B}+\gamma \bar{C}+\delta \bar{D}))=\exp \left(\begin{array}{cc}
\delta & -\beta \\
\gamma & -\delta
\end{array}\right)
$$

where $\beta, \gamma, \delta \in \mathrm{R}$. Diagonalizing, a straightforward calculation shows that

$$
\left(\begin{array}{ll}
a & b \\
c & d
\end{array}\right)=\left(\begin{array}{cc}
\cos \alpha+\delta \alpha^{-1} \sin \alpha & -\beta \alpha^{-1} \sin \alpha \\
\gamma \alpha^{-1} \sin \alpha & \cos \alpha-\delta \alpha^{-1} \sin \alpha
\end{array}\right),
$$

where $\alpha$ is the real or imaginary number such that $\alpha^{2}=\beta \gamma-\delta^{2}$ and, for imaginary $\alpha$, we understand that $\cos \alpha=\cosh i \alpha$ and $\sin \alpha=i \sinh i \alpha$. Note that, for given real $a, b, c, d$ satisfying $a d$ $-b c=1$, Eq. (19) has a solution in reals $\beta, \gamma, \delta$ if and only if $a+d \geqslant-2$. The natural action of SL on the real plane is given by

$$
\exp (-i(\beta \bar{B}+\gamma \bar{C}+\delta \bar{D}))\left(\begin{array}{l}
x \\
y
\end{array}\right)=\left(\begin{array}{ll}
a & b \\
c & d
\end{array}\right)\left(\begin{array}{l}
x \\
y
\end{array}\right)=\left(\begin{array}{l}
a x+b y \\
c x+d y
\end{array}\right) .
$$

The Euclidean special affine group $\mathrm{SA}=\mathrm{SA}(2, \mathbb{R})$ (which coincides with the Schrödinger group with one space and one time dimension) is generated by SL and the plane translates. The associated Lie algebra sa $=\mathrm{sa}(2, \mathrm{R})$ has basis $\{i \bar{B}, i \bar{C}, i \bar{D}, i \bar{P}, i \bar{Q}\}$, where

$$
\exp (-i(\mu \bar{P}+\nu \bar{Q}))\left(\begin{array}{l}
x \\
y
\end{array}\right)=\left(\begin{array}{l}
x+\mu \\
y+\nu
\end{array}\right)
$$

Evidently, $[\bar{P}, \bar{Q}]=0$. It is easy to check that the 14 other commutation relations are as in Eq. (1). We allow SA to act on $\mathcal{P}$ via the identification $(p, q)=(-y, x)$. Thus

$$
\begin{aligned}
\exp (-i(\beta \bar{B}+\gamma \bar{C}+\delta \bar{D}))\left(\begin{array}{l}
p \\
q
\end{array}\right) & =\left(\begin{array}{cc}
d & -c \\
b & a
\end{array}\right)\left(\begin{array}{l}
p \\
q
\end{array}\right), \\
\exp (-i(\mu \bar{P}+\nu \bar{Q}))\left(\begin{array}{l}
p \\
q
\end{array}\right) & =\left(\begin{array}{l}
p-\nu \\
q+\mu
\end{array}\right) .
\end{aligned}
$$

By comparing commutation relations, we see that there is a Lie algebra epimorphism hsa $\rightarrow$ sa mapping $B, C, D, P, Q, I$ to $\bar{B}, \bar{C}, \bar{D}, \bar{P}, \bar{Q}, 0$, respectively. The group epimorphism $\mathrm{HSA} \rightarrow \mathrm{SA}$ has kernel 


$$
\operatorname{Ker}(\mathrm{HSA} \rightarrow \mathrm{SA})=Z(\mathrm{HSA})=\{\exp (-i t \pi I): t \in \mathbb{R}\} .
$$

We allow HSA to act on $\mathcal{P}$ by inflation from SA. Thus

$$
\begin{gathered}
\exp (-i(\beta B+\gamma C+\delta D))\left(\begin{array}{l}
p \\
q
\end{array}\right)=\left(\begin{array}{cc}
d & -c \\
b & a
\end{array}\right)\left(\begin{array}{l}
p \\
q
\end{array}\right), \\
\exp (-i(\mu P+\nu Q+\kappa I))\left(\begin{array}{l}
p \\
q
\end{array}\right)=\left(\begin{array}{l}
p-\nu \\
q+\mu
\end{array}\right) .
\end{gathered}
$$

The state space $L^{2}(\mathbb{R})$ and the phase space plane $\mathcal{P}$ are related to each other via the continuous function

$$
\begin{gathered}
\omega: L^{2}(\mathbb{R}) \ni \psi \mapsto \omega[\psi] \in L_{\mathbb{R}}^{2}(\mathcal{P}), \\
\omega[\phi](p, q)=\frac{1}{\pi} \int_{-\infty}^{\infty} d t \overline{\phi(q+t)} \phi(q-t) \exp (2 i p t) .
\end{gathered}
$$

The function $\omega$ is essentially a specialization of the famous Weyl-Wigner correspondence; see the references at the beginning of this section, especially Refs. 19 and 18. Given $g \in$ HSA and $\psi$ $\in L^{2}(\mathrm{R})$, then

$$
\omega[\rho(g) \psi](g(p, q))=\omega[\psi](p, q) .
$$

In other words, $\omega$ is covariant with the actions of HSA on the signal space $L^{2}(R)$ and on the phase space $\mathcal{P}$. The result is proved in, for instance, Ref. 20 (Equations 6.18, 6.23, 6.27), and Ref. 18 (Proposition 2.13, Theorem 2.15). The rationale for our terminology should now be apparent: the "Heisenberg" groups HSA and HM are central extensions (or quantized versions) of the groups SA and EM.

The special linear canonical transforms are usually understood to be unitary actions of SL on the state space $L^{2}(\mathbb{R})$. For an element of SL as in Eq. (19), the action on state space is taken to be the unitary operator

$$
\lambda(a, b, c, d) \hat{U}(\beta, \gamma, \delta, 0,0,0)=\lambda(a, b, c, d) \exp (-i(\beta \hat{B}+\gamma \hat{C}+\delta \hat{D})),
$$

where $\lambda(a, b, c, d)$ is a phase. The phases $\lambda(a, b, c, d)$ cannot be chosen so as to yield a unitary representation of SL. True enough, they can be chosen so as to preserve composition up to \pm signs, thus determining a unitary representation of the metaplectic group $\operatorname{Mp}(2, R)$, which is the double cover of SL. But that observation has limited practical use, since the description of the metaplectic group is very complicated; see Ref. 18, Chap. 4. For practical purposes, the special linear canonical transforms comprise a four-dimensional group, one of the degrees of freedom being the multiplications by phases. In fact, to establish a clear correspondence with the discrete scenario, we have no choice but to include the momentum and position translates, as well as the multiplications by phases. Thus, even if one is primarily concerned with the three-parameter group SL, the connection with the discrete scenario demands that we consider all six degrees of freedom in the group HSA.

\section{DISCRETE AFFINE CANONICAL TRANSFORMS}

We shall introduce some discrete affine canonical transforms whose infinitesmal generators satisfy matrix equations analogous to Eqs. (11)-(17). First, we need to look at the Kravchuk functions, which are discrete analogs of the Hermite functions. We closely follow the representation-theoretic discussion of the Kravchuk functions in Ref. 24 (Chap. 6) and, to a lesser extent, Ref. 25 (Chap. 8). For parallel discussions of the Kravchuk and Hermite functions in 
connection with discrete and continuum oscillator algebras, see Refs. 10 and 26. An alternative approach to the comparison of Kravchuk and Hermite functions, making systematic use of creation and annihilation operators, can be found in Ref. 27.

All lemmas that we state without proof can be obtained from the earlier lemmas together with routine calculations as in Ref. 24. There is only one argument that is not straightforward, namely, the proof of Lemma 4.5. For this, Ref. 24 invokes the theory of hypergeometric functions, and that requires some delicate analysis, the Kravchuk functions being specializations of hypergeometric functions at singular points. Our more direct argument is purely algebraic. The results proved below concerning Kravchuk functions and Kravchuk polynomials are summarized in Appendix B.

Let $n$ be a positive integer. Write $n=2 \ell+1$. Let $[n]$ denote the set of $k$ such that $\ell+k$ and $\ell-k$ are natural numbers. Thus, $[n]$ consists of $n$ integers or $n$ halves of odd integers. Let $\mathcal{L}_{n}$ be the $n$-dimensional Hilbert space of functions $[n] \rightarrow \mathrm{C}$, the inner product being

$$
\langle\psi \mid \chi\rangle=\sum_{k=-\ell}^{\ell} \overline{\psi(k)} \chi(k)
$$

where $\psi, \chi \in \mathcal{L}_{n}$, and the bar denotes complex conjugation. Let $|k\rangle_{n}^{Z}$ denote the vector in $\mathcal{L}_{n}$ such that, given $\psi \in \mathcal{L}_{n}$, then $\psi(k)=\langle\psi \mid k\rangle_{n}^{Z}$. The set $\left\{|k\rangle_{n}^{Z}: k \in[n]\right\}$ is an orthonormal basis for $\mathcal{L}_{n}$. Via the equation

$$
|k\rangle_{n}^{Z}=\frac{u^{\ell+k} v^{\ell-k}}{\sqrt{(\ell+k) !(\ell-k) !}}
$$

we identify $\mathcal{L}_{n}$ with the space of homogenous polynomials of degree $2 \ell$ in variables $u$ and $v$.

Later, we shall be realizing $\mathcal{L}_{n}$ as a representation space of the Lie group $\mathrm{U}(2)$. For the following three preliminary results, though, we may as well consider, more generally, the Lie group GL(2,C). We define a group representation $\rho_{n}$ of GL(2,C) on $\mathcal{L}_{n}$ such that

$$
\left(\rho_{n}(g) F\right)(u, v)=F(a u+c v, b u+d v), \quad g=\left(\begin{array}{ll}
a & b \\
c & d
\end{array}\right) .
$$

Lemma 4.1: Let $j, k \in[n]$. Put $\max =\max (0, j+k)$ and $\min =\min (\ell+j, \ell+k)$. Then, with respect to the orthonormal basis $\left\{|k\rangle_{n}^{Z}: k \in[n]\right\}$, the $(j, k)$ entry of the matrix representing $\rho_{n}(g)$ is

$$
{ }_{n}^{Z}\left\langle j\left|\rho_{n}(g)\right| k\right\rangle_{n}^{Z}=\sqrt{\frac{(\ell+j) !(\ell-j) !}{(\ell+k) !(\ell-k) !}} \sum_{r=\max }^{\min }\left(\begin{array}{c}
\ell+k \\
r
\end{array}\right)\left(\begin{array}{c}
\ell-k \\
\ell+j-r
\end{array}\right) a^{r} b^{\ell+j-r} c^{\ell+k-r} d^{r-j-k} .
$$

Henceforth, we work directly from Lemma 4.1, and we can forget about the characterization of $\mathcal{L}_{n}$ as a space of polynomials.

Lemma 4.2: Now suppose that $g \in \mathrm{SL}(2, \mathrm{C})$, and that the matrix entries $b, c, d$ are nonzero. Given $j, k \in[n]$, then

$$
{ }_{n}^{Z}\left\langle j\left|\rho_{n}(g)\right| k\right\rangle_{n}^{Z}=\frac{b^{\ell+j} c^{\ell+k}}{d^{j+k}} \sqrt{\frac{(\ell+j) !(\ell+k) !}{(\ell-j) !(\ell-k) !}} \sum_{r=0}^{\min (\ell+j, \ell+k)} \frac{(2 \ell-r) !(b c)^{-r}}{\nu !(\ell+j-r) !(\ell+k-r) !} .
$$

Let $c_{r}=\ell(\ell+1)-\kappa^{2}+\frac{1}{4}$ for $2 \kappa \in \mathbb{Z}$. Thus

$$
c_{k+1 / 2}=(\ell-k)(\ell+k+1), \quad c_{k-1 / 2}=(\ell+k)(\ell-k+1) .
$$

Let $\sigma_{n}$ be the differential representation of $\rho_{n}$.

Lemma 4.3: Given an element $H=\left(\begin{array}{cc}A & B \\ C & D\end{array}\right)$ of $\mathrm{gl}(2, \mathrm{C})$ and an element $k \in[n]$, then

$$
\sigma_{n}(H)|k\rangle_{n}^{Z}=\sqrt{c_{k-1 / 2}} C|k-1\rangle_{n}^{Z}+((\ell+k) A+(\ell-k) D)|k\rangle_{n}^{Z}+\sqrt{c_{k+1 / 2}} B|k+1\rangle_{n}^{Z} .
$$


The real Lie algebra $\mathrm{u}(2)$ and its subalgebra su(2) have bases $\{-i W,-i X,-i Y,-i Z\}$ and $\{-i X,-i Y,-i Z\}$, respectively, where

$$
W=\frac{1}{2}\left(\begin{array}{cc}
1 & 0 \\
0 & 1
\end{array}\right), \quad X=\frac{1}{2}\left(\begin{array}{cc}
0 & 1 \\
1 & 0
\end{array}\right), \quad Y=\frac{1}{2}\left(\begin{array}{cc}
0 & -i \\
i & 0
\end{array}\right), \quad Z=\frac{1}{2}\left(\begin{array}{cc}
1 & 0 \\
0 & -1
\end{array}\right) .
$$

Note that $W$ commutes with $X, Y, Z$, and the other commutation relations are $[X, Y]=i Z$ and $[Y, Z]=i X$ and $[Z, X]=i Y$. Let

$$
\hat{W}_{n}=\sigma_{n}(W), \quad \hat{X}_{n}=\sigma_{n}(X), \quad \hat{Y}_{n}=\sigma_{n}(Y), \quad \hat{Z}_{n}=\sigma_{n}\left(Z_{n}\right) .
$$

Given $k \in[n]$, then, by Lemma 4.3,

$$
\begin{gathered}
\hat{W}_{n}|k\rangle_{n}^{Z}=\ell|k\rangle_{n}^{Z}, \\
\hat{X}_{n}|k\rangle_{n}^{Z}=\frac{1}{2}\left(\sqrt{c_{k-1 / 2}}|k-1\rangle_{n}^{Z}+\sqrt{c_{k+1 / 2}}|k+1\rangle_{n}^{Z}\right), \\
\hat{Y}_{n}|k\rangle_{n}^{Z}=\frac{i}{2}\left(\sqrt{c_{k-1 / 2}}|k-1\rangle_{n}^{Z}-\sqrt{c_{k+1 / 2}}|k+1\rangle_{n}^{Z}\right), \\
\hat{Z}_{n}|k\rangle_{n}^{Z}=k|k\rangle_{n}^{Z} .
\end{gathered}
$$

Thus, the algebra representation $\sigma_{n}$ of $\mathrm{gl}(2, \mathrm{C})$ restricts to anti-Hermitian representations of $\mathrm{u}(2)$ and isu(2). In other words, the group representation $\rho_{n}$ of GL(2,C) restricts to unitary representations of $\mathrm{U}(2)$ and $\mathrm{SU}(2)$. It is well-known (by an easy ladder argument) that the two restricted representations are irreducible.

For each $k \in[n]$, we define a vector

$$
|k\rangle_{n}^{X}=\exp \left(-i \pi \hat{Y}_{n} / 2\right)|k\rangle_{n}^{Z} .
$$

To rewrite Eqs. (26)-(29) with respect to the orthonormal basis $\left\{|k\rangle_{n}^{X}: k \in[n]\right\}$, let us first determine the exponentials of $i W, i X, i Y, i Z$. By evaluating derivatives at $t=0$, or by appealing to Eq. (19) (with complex values of $\beta, \gamma, \delta$ ), we have

$$
\begin{gathered}
\exp (-i t W)=\left(\begin{array}{cc}
e^{-i t / 2} & 0 \\
0 & e^{-i t / 2}
\end{array}\right), \quad \exp (-i t X)=\left(\begin{array}{cc}
\cos t / 2 & -i \sin t / 2 \\
-i \sin t / 2 & \cos t / 2
\end{array}\right), \\
\exp (-i t Y)=\left(\begin{array}{cc}
\cos t / 2 & -\sin t / 2 \\
\sin t / 2 & \cos t / 2
\end{array}\right), \quad \exp (-i t Z)=\left(\begin{array}{cc}
e^{-i t / 2} & 0 \\
0 & e^{i t / 2}
\end{array}\right) .
\end{gathered}
$$

By direct calculation, $e^{-i t Y} Z e^{i t Y}=Z \cos t+X \sin t$ for all $t \in \mathbb{R}$. So

$$
\exp \left(-i \pi \hat{Y}_{n} / 2\right) \hat{Z}_{n} \exp \left(i \pi \hat{Y}_{n} / 2\right)=\hat{X}_{n}, \quad \exp \left(-i \pi \hat{Y}_{n} / 2\right) \hat{X}_{n} \exp \left(i \pi \hat{Y}_{n} / 2\right)=-\hat{Z}_{n} .
$$

We can now rewrite Eqs. (26)-(29) as

$$
\begin{gathered}
\hat{W}_{n}|k\rangle_{n}^{X}=\ell|k\rangle_{n}^{X}, \\
\hat{X}_{n}|k\rangle_{n}^{X}=k|k\rangle_{n}^{X}, \\
\hat{Y}_{n}|k\rangle_{n}^{X}=\frac{i}{2}\left(\sqrt{c_{k-1 / 2}}|k-1\rangle_{n}^{X}-\sqrt{c_{k+1 / 2}}|k+1\rangle_{n}^{X}\right),
\end{gathered}
$$




$$
\hat{Z}_{n}|k\rangle_{n}^{X}=\frac{-1}{2}\left(\sqrt{c_{k-1 / 2}}|k-1\rangle_{n}^{X}+\sqrt{c_{k+1 / 2}}|k+1\rangle_{n}^{X}\right) .
$$

Lemmas 4.1 and 4.2 now yield the following result.

Lemma 4.4: Given $j, k \in[n]$, then

$$
\begin{aligned}
& \text { (1) }{ }_{n}^{Z}\langle j \mid k\rangle_{n}^{X}=\frac{(-1)^{\ell+j}}{2^{\ell}} \sqrt{\frac{(\ell+j) !(\ell-j) !}{(\ell+k) !(\ell-k) !}} \sum_{r}\left(\begin{array}{c}
\ell+k \\
r
\end{array}\right)\left(\begin{array}{c}
\ell-k \\
\ell+j-r
\end{array}\right)(-1)^{r}, \\
& \text { (2) }{ }_{n}^{Z}\langle j \mid k\rangle_{n}^{X}=\frac{(-1)^{\ell+j}}{2^{\ell}} \sqrt{\frac{(\ell+j) !(\ell+k) !}{(\ell-j) !(\ell-k) !}} \sum_{s} \frac{(2 \ell-s) !(-2)^{s}}{s !(\ell+j-\nu) !(\ell+k-s) !},
\end{aligned}
$$

where the indices of the sums run over the values for which the terms are defined, namely, $\max (0, j+k) \leqslant r \leqslant \min (\ell+j, \ell+k)$ and $0 \leqslant s \leqslant \min (\ell+j, \ell+k)$.

Lemma 4.5: Given $j, k \in[n]$, then

$$
{ }_{n}^{Z}\langle k \mid j\rangle_{n}^{X}=(-1)^{j-k} \quad{ }_{n}^{Z}\langle j \mid k\rangle_{n}^{X}=(-1)^{\ell+k} \quad{ }_{n}^{Z}\langle k \mid-j\rangle_{n}^{X}=(-1)^{\ell-j} \quad{ }_{n}^{Z}\langle k \mid j\rangle_{n}^{X} .
$$

Proof: Throughout the argument, when multiplying powers of -1 , we must bear in mind that $j, k, \ell$ are all integers or all halves of odd integers. By Lemma 4.4(2),

$$
(-1)^{\ell+j} \quad{ }_{n}^{Z}\langle j \mid k\rangle_{n}^{X}=(-1)^{\ell+k} \quad{ }_{n}^{Z}\langle k \mid j\rangle_{n}^{X} .
$$

The first asserted equality follows.

Since the eigenvalues of $\hat{X}_{n}$ are distinct, the eigenvector equations $\hat{X}_{n}|j\rangle_{n}^{X}=|j\rangle_{n}^{X}$ and $\hat{X}_{n} \mid$ $-j\rangle_{n}^{X}=-j|j\rangle_{n}^{X}$ determine the unit vectors $|j\rangle_{n}^{X}$ and $|-j\rangle_{n}^{X}$ up to phase factors. By Eqs. (27) and (33), the matrix entry ${ }_{n}^{Z}\left\langle j\left|\hat{X}_{n}\right| k\right\rangle_{n}^{X}$ is zero unless $|j-k|=1$. Therefore, fixing $j$, there is a phase $\omega$ such that, for all $k$, we have

$$
{ }_{n}^{Z}\langle k \mid-j\rangle_{n}^{X}=\omega(-1)^{\ell+k} \underset{n}{Z}\langle k \mid j\rangle_{n}^{X} .
$$

(In other words, if we multiply the $Z$-coordinates of $|j\rangle_{n}^{X}$ by an alternating \pm 1 , then we get a multiple of $|-j\rangle_{n}^{X}$.) Putting $k=-\ell$, and noting that, by Lemma 4.4(1),

$$
{ }_{n}^{Z}\langle-\ell \mid-j\rangle_{n}^{X}=\frac{1}{2} \sqrt{\left(\begin{array}{c}
2 \ell \\
\ell+j
\end{array}\right)} \quad{ }_{n}^{Z}\langle-\ell \mid j\rangle_{n}^{X},
$$

we deduce that $\omega=1$. The second asserted equality follows and, hence, the third.

Lemma 4.6: Given $j, k \in[n]$, then

$$
\begin{array}{lll}
\text { (1) } \sqrt{c_{k-1 / 2}}{ }_{n}^{Z}\langle k-1 \mid j\rangle_{n}^{X}-2 j_{n}^{Z}\langle k \mid j\rangle_{n}^{X}+\sqrt{c_{k+1 / 2}} & { }_{n}^{Z}\langle k+1 \mid j\rangle_{n}^{X}=0, \\
\text { (2) } \sqrt{c_{j-1 / 2}} \quad{ }_{n}^{Z}\langle k \mid j-1\rangle_{n}^{X}-2 k_{n}^{Z}\langle k \mid j\rangle_{n}^{X}+\sqrt{c_{j+1 / 2}}{ }_{n}^{Z}\langle k \mid j+1\rangle_{n}^{X}=0 .
\end{array}
$$

Let $\mathbb{N}_{n}$ denote the set of natural numbers less than $n$. For each $s \in \mathbb{N}_{n}$, we define the Kravchuk polynomial $K_{s, n}: \mathbb{N}_{n} \rightarrow \mathrm{C}$ and the Kravchuk function $h_{s, n}:[n] \rightarrow \mathrm{C}$ such that

$$
\frac{(-1)^{\ell+j}}{2^{\ell}} \sqrt{\left(\begin{array}{c}
2 \ell \\
\ell+j
\end{array}\right)\left(\begin{array}{c}
2 \ell \\
\ell+k
\end{array}\right)} K_{\ell+j, n}(\ell+k)=h_{\ell+j, n}(k)={ }_{n}^{Z}\langle j \mid k\rangle_{n}^{X}
$$

for $j, k \in[n]$. The formulas in Appendix B are precisely Lemmas 4.4-4.6.

Proposition 4.7: The set of Kravchuk functions $\left\{h_{s, n}: s \in \mathbb{N}_{n}\right\}$ is an orthonormal basis for $\mathcal{L}_{n}$.

Proof: The values of the Kravchuk functions are the overlaps of two orthonormal bases.

We now rewrite the Kravchuk functions as $|s\rangle_{n}=h_{s, n}$. 
Proposition 4.8: Given $s \in[n]$, then $|s\rangle_{n}=(-1)^{s}|\ell-s\rangle_{n}^{X}$.

Proof: Apply Lemma 4.5.

Via Proposition 4.8, we can rewrite Eqs. (32)-(35) as

$$
\begin{gathered}
\hat{W}_{n}|s\rangle_{n}=\ell|s\rangle_{n}, \\
\hat{X}_{n}|s\rangle_{n}=(\ell-s)|s\rangle_{n}, \\
\hat{Y}_{n}|s\rangle_{n}=\frac{i}{2}\left(-\sqrt{s(2 \ell+1-s)}|s-1\rangle_{n}+\sqrt{(s+1)(2 \ell-s)}|s+1\rangle_{n}\right), \\
\hat{Z}_{n}|s\rangle_{n}=\frac{1}{2}\left(\sqrt{s(2 \ell+1-s)}|s-1\rangle_{n}+\sqrt{(s+1)(2 \ell-s)}|s+1\rangle_{n}\right) .
\end{gathered}
$$

We define Hermitian operators

$$
\begin{gathered}
\hat{I}_{n}=\hat{X}_{n} / \ell, \quad \hat{P}_{n}=-\hat{Y}_{n} / \sqrt{\ell}, \quad \hat{Q}_{n}=\hat{Z}_{n} / \sqrt{\ell}, \\
2 \hat{B}_{n}=\hat{P}_{n}^{2}, \quad 2 \hat{C}_{n}=\hat{Q}_{n}^{2}, \quad 2 \hat{D}_{n}=\hat{P}_{n} \hat{Q}_{n}+\hat{Q}_{n} \hat{P}_{n} .
\end{gathered}
$$

We can understand $\hat{P}_{n}$ as discrete momentum (or frequency) and $\hat{Q}_{n}$ as discrete position (or time). For real $\beta, \gamma, \delta, \mu, \nu, \kappa$, we introduce a Hermitian operator

$$
\hat{H}_{n}(\beta, \gamma, \delta, \mu, \nu, \kappa)=\beta \hat{B}_{n}+\gamma \hat{C}_{n}+\delta \hat{D}_{n}+\mu \hat{P}_{n}+\nu \hat{Q}_{n}+\kappa \hat{I}_{n} .
$$

We define a discrete affine canonical transform to be a unitary operator having the form

$$
\hat{U}_{n}(\beta, \gamma, \delta, \mu, \nu, \kappa)=\exp \left(-i \hat{H}_{n}(\beta, \gamma, \delta, \mu, \nu, \kappa)\right) .
$$

Recall that, in the continuum scenario, we defined the continuum affine canonical transforms to be the composites of the unitary operators having the form $\hat{U}(\beta, \ldots, \kappa)$. Our reason for not defining the discrete affine canonical transforms in the same way is that the infinitesmal generators $\hat{H}_{n}(\beta, \ldots, \kappa)$ do not span a Lie algebra. We can work with single-parameter groups of discrete affine canonical transforms-including fractional Fourier transforms, chirps and dilations-and these single-parameter groups, of course, have the index-additivity property $\hat{U}^{s} \hat{U}^{t}=\hat{U}^{s+t}$. In general, though, we do not retain any tractible closure property if we compose elements of distinct single-parameter groups.

However, in the continuum scenario, we defined the motion canonical transforms to be precisely the unitary operators having the form $\hat{E}(\kappa, \lambda, \mu, \nu)$, these operators being closed under composition. That feature can be retained in the discrete scenario. Let

$$
\hat{N}_{n}=\hat{W}_{n}-\hat{X}_{n}=\ell\left(\hat{1}-\hat{I}_{n}\right) .
$$

The operators $\hat{I}_{n}, \hat{N}_{n}, \hat{P}_{n}, \hat{Q}_{n}$ are closed under commutators. We define a discrete motion canonical transform to be a unitary operator having the form

$$
\hat{E}_{n}(\kappa, \lambda, \mu, \nu)=\exp \left(-i\left(\kappa \hat{I}_{n}+\lambda \hat{N}_{n}+\mu \hat{P}_{n}+\nu \hat{Q}_{n}\right)\right)=\rho\left(E_{n}(\kappa, \lambda, \mu, \nu)\right),
$$

where $\kappa, \lambda, \mu, \nu \in \mathbb{R}$. Let us put it in the language of representations. The Lie group $\mathrm{u}(2)$ has a basis $\left\{I_{n}, N_{n}, P_{n}, Q_{n}\right\}$ where

$$
I_{n}=X / \ell, \quad N_{n}=W-X, \quad P_{n}=-Y / \sqrt{\ell}, \quad Q_{n}=Z / \sqrt{\ell} .
$$

The commutation relations are 


$$
\begin{aligned}
& {\left[I_{n}, N_{n}\right]=0, \quad\left[I_{n}, P_{n}\right]=-i Q_{n} / \ell, \quad\left[I_{n}, Q_{n}\right]=i P_{n} / \ell,} \\
& {\left[N_{n}, P_{n}\right]=i Q_{n}, \quad\left[N_{n}, Q_{n}\right]=-i P_{n}, \quad\left[P_{n}, Q_{n}\right]=i I_{n} .}
\end{aligned}
$$

The algebra representation $\sigma_{n}$ maps $I_{n}, N_{n}, P_{n}, Q_{n}$ to $\hat{I}_{n}, \hat{N}_{n}, \hat{P}_{n}, \hat{Q}_{n}$, respectively. Observe that, as $\ell \rightarrow \infty$, the structural constants for $I_{n}, N_{n}, P_{n}, Q_{n}$ converge to those given in Sec. 2 for the basis elements $I, N, P, Q$ of $\mathrm{hm}$. The algebra iu(2) and the group $\mathrm{U}(2)$ are to serve as the discrete analogs of the algebra $\mathrm{hm}$ and the group HM.

Now let us write down the matrices for $\hat{B}_{n}, \hat{C}_{n}, \hat{D}_{n}, \hat{P}_{n}, \hat{Q}_{n}, \hat{N}_{n}, \hat{I}_{n}$ with respect to the basis of Kravchuk functions. For $2 r+1 \in \mathbb{N}$, let

$$
t_{n}(r)=\sqrt{(2 r+1)(4 \ell-2 r+1) / 16 \ell} .
$$

Given $s \in \mathbb{N}_{n}$, then

$$
t_{n}\left(s+\frac{1}{2}\right)=\sqrt{\frac{s+1}{2}\left(1-\frac{s}{2 \ell}\right)}, \quad t_{n}\left(s-\frac{1}{2}\right)=\sqrt{\frac{s}{2}\left(1-\frac{s-1}{2 \ell}\right)} .
$$

By Eqs. (36)-(39),

$$
\begin{gathered}
\hat{B}_{n}|s\rangle_{n}=-\frac{1}{2} t_{n}\left(s-\frac{1}{2}\right) t_{n}\left(s-\frac{3}{2}\right)|s-2\rangle_{n}+\left(\frac{s}{2}\left(1-\frac{s}{2 \ell}\right)+\frac{1}{4}\right)|s\rangle_{n} \\
-\frac{1}{2} t_{n}\left(s+\frac{1}{2}\right) t_{n}\left(s+\frac{3}{2}\right)|s+2\rangle_{n} \\
\hat{C}_{n}|s\rangle_{n}=\frac{1}{2} t_{n}\left(s-\frac{1}{2}\right) t_{n}\left(s-\frac{3}{2}\right)|s-2\rangle_{n}+\left(\frac{s}{2}\left(1-\frac{s}{2 \ell}\right)+\frac{1}{4}\right)|s\rangle_{n}+\frac{1}{2} t_{n}\left(s+\frac{1}{2}\right) t_{n}\left(s+\frac{3}{2}\right)|s+2\rangle_{n}, \\
\hat{D}_{n}|s\rangle_{n}=-i t_{n}\left(s-\frac{1}{2}\right) t_{n}\left(s-\frac{3}{2}\right)|s-2\rangle_{n}+i t_{n}\left(s+\frac{1}{2}\right) t_{n}\left(s+\frac{3}{2}\right)|s+2\rangle_{n} \\
\hat{P}_{n}|s\rangle_{n}=-i t_{n}\left(s-\frac{1}{2}\right)|s-1\rangle_{n}+i t_{n}\left(s+\frac{1}{2}\right)|s+1\rangle_{n} \\
\hat{Q}_{n}|s\rangle_{n}=t_{n}\left(s-\frac{1}{2}\right)|s-1\rangle_{n}+t_{n}\left(s+\frac{1}{2}\right)|s+1\rangle_{n} \\
\hat{I}_{n}|s\rangle_{n}=(1-s / \ell)|s\rangle_{n} \\
\hat{N}_{n}|s\rangle_{n}=s|s\rangle_{n} .
\end{gathered}
$$

Again, we observe a suggestive connection with the continuum scenario. As $\ell \rightarrow \infty$, the matrix entries in Eqs. (44)-(50) converge to the matrix entries in Eqs. (11)-(17).

In Sec. II, we ended with an example. Let us end the present section with the analogous example. The discrete Fourier transform of Atakishiyev-Wolf ${ }^{10}$ is the unitary operator $\hat{F}_{n}$ on $\mathcal{L}_{n}$ such that $\hat{F}_{n}|s\rangle_{n}=i^{s}|s\rangle_{n}$. More generally, their discrete fractional Fourier transform of order $t$ $\in \mathbb{R}$ is the unitary operator $\hat{F}_{n}^{t}$ on $\mathcal{L}_{n}$ such that $\hat{F}_{n}^{t}|s\rangle_{n}=\exp (2 \pi i s t)|s\rangle_{n}$. In other words,

$$
\hat{F}_{n}^{t}=\exp \left(2 \pi i t \hat{N}_{n}\right)
$$

\section{CONVERGENCE OF UNITARY TRANSFORMS}

We wish to say that the continuum affine canonical transforms are limits of discrete affine canonical transforms. The whole problem lies in making the assertion absolutely unambiguous; then the proof will follow purely by deductive reasoning. Parts of the proof are deferred to Ref. 4. 
Usually, when one writes an equation of the form $x=\lim _{n \rightarrow \infty} x_{n}$, the object $x$ and the objects $x_{n}$ all belong to the same space (or category). Such is not the case in our situation. We need to specify an interface between the continuum scenario and the discrete scenario. Let us describe the interface in two different ways, the first one clear and precise, the second one more illuminating from a practical perspective.

The clear description of the interface makes use of inductive limits, which are introduced in Refs. 1-3. A summary is given in Ref. 4, Sec. 2. Let $\mathcal{S}(\mathbb{R})$ be the Schwartz subspace of $L^{2}(\mathbb{R})$. For each positive integer $n$, let res $_{n}$ be the linear map $\mathcal{S}(\mathbb{R}) \rightarrow \mathcal{L}_{n}$ such that, given $\phi \in \mathcal{S}(\mathbb{R})$, and writing $\phi_{n}=\operatorname{res}_{n}(\phi)$, then

$$
\phi_{n}(k)=\ell^{-1 / 4} \phi\left(\ell^{-1 / 2} k\right)
$$

where $k \in[n]$. The linear maps res ${ }_{n}$ comprise an inductive resolution of $L^{2}(\mathbb{R})$. We are now in a position to realize vectors $\psi$ in $L^{2}(\mathbb{R})$ as limits $\psi=\lim _{n} \psi_{n}$, where each $\psi_{n}$ is a vector in the $n$-dimensional space $\mathcal{L}_{n}$. We can do the same for bounded operators and, in particular, for unitary operators.

The following alternative description is rather more intuitive. Let $\phi$ be a continuous and well-behaved complex-valued function with one real variable. For each $n$, let $\phi_{n}$ be a vector in $\mathcal{L}_{n}$. We regard $\phi_{n}$ as a good approximation to $\phi$ provided

$$
\phi_{n}(k) \approx \ell^{-1 / 4} \phi\left(\ell^{-1 / 2} k\right)
$$

for almost all $k \in[n]$. As the number of sample points $n=2 \ell+1$ increases, the mesh $\ell^{-1 / 2}$ decreases and the width of the sample window $2 \ell^{1 / 2}$ increases. If $\phi_{n}$ becomes an arbitrarily good approximation to $\phi$ in a certain manner that preserves everything involving inner products, then we say that $\phi_{n}$ converges to $\phi$, and we write $\phi=\lim _{n} \phi_{n}$. Limits of unitary operators are required to preserve limits of vectors.

For example, Ref. 2, Theorem 5.1, says that

$$
|s\rangle=\lim _{n}|s\rangle_{n},
$$

for all natural numbers $s$. In other words, the Hermite functions are the inductive limits of the Kravchuk functions.

Theorem 5.1: Let $\beta=\lim _{n} \beta_{n}, \quad \gamma=\lim _{n} \gamma_{n}, \quad \delta=\lim _{n} \delta_{n}, \quad \mu=\lim _{n} \mu_{n}, \quad \nu=\lim _{n} \nu_{n}, \quad \kappa$ $=\lim _{n} \kappa_{n}$ as limits of real sequences. Then

$$
\hat{U}(\beta, \gamma, \delta, \mu, \nu, \kappa)=\lim _{n} \hat{U}_{n}\left(\beta_{n}, \gamma_{n}, \delta_{n}, \mu_{n}, \nu_{n}, \kappa_{n}\right) .
$$

Proof: This is part of Ref. 4, Theorem 7.2.

A comparison of Eqs. (11)-(16) with Eqs. (44)-(49) provides a heuristic justification for Theorem 5.1, but not a proof. Convergence of matrix entries of infinitesmal generators does not, in general, imply convergence of the corresponding unitary operators.

Although arbitrary pairs of discrete affine canonical transforms do not compose in a tractible way, let us draw attention to the index-additivity property of single-parameter groups of discrete affine canonical transforms. Fix reals $\beta, \gamma, \delta, \mu, \nu, \kappa$. Theorem 5.1 tells us that

$$
\hat{U}(t \beta, t \gamma, t \delta, t \mu, t \nu, t \kappa)=\lim _{n} \hat{U}_{n}\left(t \beta_{n}, t \gamma_{n}, t \delta_{n}, t \mu_{n}, t \nu_{n}, t \kappa_{n}\right)
$$

for all $t \in \mathrm{R}$. Since $\hat{B}_{n}, \hat{C}_{n}, \hat{D}_{n}, \hat{P}_{n}, \hat{Q}_{n}, \hat{I}_{n}$ are linearly independent for $n \geqslant 3$, we have the following.

Corollary 5.2: For fixed $n \geqslant 3$, Eq. (54) describes a bijective correspondence between the single-parameter groups of continuum affine canonical transforms and the single-parameter 
groups of discrete affine canonical transforms on $\mathcal{L}_{n}$. Now let $n$ vary. The elements of a singleparameter group of continuum affine canonical transforms are inductive limits of sequences of elements of the corresponding single-parameter groups of discrete affine canonical transforms.

We now turn to motion canonical transforms.

Theorem 5.3: Let $\kappa=\lim _{n} \kappa_{n}, \lambda=\lim _{n} \lambda_{n}, \mu=\lim _{n} \mu_{n}, \nu=\lim _{n} \nu_{n}$ as limits of real sequences. Then

$$
\hat{E}(\kappa, \lambda, \beta, \gamma)=\lim _{n} \hat{E}_{n}\left(\kappa_{n}, \lambda_{n}, \beta_{n}, \delta_{n}\right) .
$$

Proof: The limit of representations in Ref. 4, Theorem 10.2, is a stronger result.

Warning: Theorem 5.3 is not a special case of Theorem 5.1. Not all of the discrete motion canonical transforms are discrete affine canonical transforms.

Comparing Eqs. (18) and (51), we see that Theorem 5.3 recovers the convergence of fractional Fourier transforms

$$
\hat{F}^{t}=\lim _{n} \hat{F}_{n}^{t}
$$

A more direct proof of Eq. (55) is given in Ref. 3, Example 4.F. The equation (not expressed in the form of an inductive limit) is due to Atakishiyev-Wolf. ${ }^{10}$

\section{COMPLEX-ORDER FOURIER TRANSFORMS AND COHERENT STATES}

We introduce two more objects to the continuum scenario: the system of Glauber coherent states (Gabor functions) and the continuum Hermite semigroup (the semigroup of complex-order Fourier transforms). Then we introduce the analogous objects to the discrete scenario: the system of spin coherent states and the discrete Hermite semigroup (discrete complex-order Fourier transforms). As in the previous section, the analogy between the discrete and continuum objects is plain enough; our purpose is to express the analogy precisely using inductive limits.

For an introduction to the Glauber and spin coherent states, see Ref. 28, Chap. 1 or Ref. 29. To fix notation, we shall recall the relevant definitions, but we shall not discuss the measures on the label spaces. The Glauber coherent state $|z\rangle^{C}$ with label $z \in \mathbb{C}$ can be defined as

$$
|z\rangle^{C}=\exp \left(-|z|^{2} / 2\right) \exp \left(z \hat{A}^{\dagger}\right)|0\rangle=\exp \left(-|z|^{2} / 2\right) \sum_{s=0}^{\infty} \frac{z^{s}}{\sqrt{s !}}|s\rangle .
$$

Writing $g_{z}$ to denote $|z\rangle^{C}$ regarded as a (rapidly decreasing) function $\mathrm{R} \rightarrow \mathrm{C}$, we have

$$
\pi^{1 / 4} g_{z}(q)=\exp \left(-\frac{q^{2}}{2}+\sqrt{2} z q-\frac{z^{2}}{2}-\frac{|z|^{2}}{2}\right)=\exp \left(-\frac{q^{2}}{2}-(u+i v) q-\frac{u^{2}}{2}-\frac{i u v}{2}\right),
$$

where $\sqrt{2} z=u+i v$ with $u, v \in \mathbb{R}$. We note one other useful characterization:

$$
\left.|z\rangle^{C}=\exp (-i u \hat{P}+i v \hat{Q})\right)|0\rangle .
$$

In electrical enginnering and signal processing, Glauber coherent states are usually called Gabor functions, and are usually expressed in the form of Eq. (57). The other two equations are more normally used in quantum physics. As a gesture of mediation between the two disciplines, let us give a quick proof that the three equations are mutually equivalent. From Eq. (58), rewritten as

$$
g_{z}=\exp (-i u \hat{P}+i v \hat{Q}) h_{0},
$$

it is easy to obtain Eq. (57) using the identities 


$$
\begin{gathered}
\exp (-i(u \hat{P}+v \hat{Q}))=\exp (i u v / 2) \exp (i v \hat{Q}) \exp (-i u \hat{P}), \\
\exp (-i u \hat{P}) \phi(q)=\phi(q-u), \quad \exp (-i v \hat{Q}) \phi(q)=\exp (i v q) \phi(q),
\end{gathered}
$$

where $\phi \in \mathcal{S}(\mathrm{R})$. Using the generating function

$$
\exp \left(2 q t-t^{2}\right)=\sum_{s=0}^{\infty} \frac{t^{s}}{s !} H_{s}(q)
$$

together with Eq. (57), straightforward manipulation yields

$$
\sum_{s=0}^{\infty} \frac{t^{s}}{s !} \int_{-\infty}^{\infty} d q H_{s}(q) \exp \left(-q^{2} / 2\right) g_{z}(q)=\pi^{1 / 4} \exp \left(-|z|^{2} / 2\right) \exp (\sqrt{2} z t) .
$$

Comparing coefficients of powers of $t$, we obtain $\langle s \mid z\rangle^{C}=\exp \left(-|z|^{2} / 2\right) / \sqrt{s !}$. The equivalence of Eqs. (56)-(58) is now established.

For $\zeta \in \mathrm{C}$ with $|\zeta| \leqslant 1$, the continuum complex-order Fourier transform $\hat{F}(\zeta)$ is defined to be the bounded operator on $L^{2}(\mathbb{R})$ such that

$$
\hat{F}(\zeta)|s\rangle=\zeta^{s}|s\rangle .
$$

The integral kernel for $\hat{F}(\zeta)$ may be found in Ref. 30. An optical realization of $\hat{F}(\zeta)$ is discussed in Ref. 31. We have an obvious composition law

$$
\hat{F}(\zeta) \hat{F}\left(\zeta^{\prime}\right)=\hat{F}\left(\zeta \zeta^{\prime}\right) .
$$

The commutative semigroup $\{\hat{F}(\zeta):|\zeta| \leqslant 1\}$, called the continuum Hermite semigroup, is evidently isomorphic to the semigroup $\left\{\zeta \in C_{0}|\zeta| \leqslant 1\right\}$. Writing

$$
\zeta=\exp (2 \pi i t)
$$

we say that $\hat{F}(\zeta)$ has order $t$. Given $\hat{F}(\zeta)$, the real part of $t$ is well-defined up to congruence modulo 1 . The condition $|\xi| \leqslant 1$ is precisely the condition that $t$ lies in the closed upper half of the complex plane. By Eq. (17),

$$
\hat{F}(\zeta)=\exp (2 \pi i t \hat{N})
$$

The continuum fractional Fourier transforms are precisely the unitary continuum complex-order Fourier transforms. By Eqs. (56) and (59), the continuum Hermite semigroup permutes the Glauber coherent states (up to scalar factors) according to the equation

$$
\hat{F}(\zeta)|z\rangle^{C}=\exp \left(|z \zeta|^{2} / 2-|z|^{2} / 2\right)|\zeta z\rangle^{C} .
$$

Now let us look at the discrete scenario. The discrete annihilation operator $\hat{A}_{n}$ and its Hermitian conjugate, the discrete creation operator $\hat{A}_{n}^{\dagger}$, are defined to be

$$
\hat{A}_{n}=\left(\hat{Q}_{n}+i \hat{P}_{n}\right) / \sqrt{2}, \quad \hat{A}_{n}^{\dagger}=\left(\hat{Q}_{n}-i \hat{P}_{n}\right) / \sqrt{2} .
$$

From Eqs. (47) and (48) we have

$$
\hat{A}_{n}|s\rangle_{n}=\sqrt{s\left(1-\frac{s-1}{2 \ell}\right)}|s-1\rangle_{n}, \quad \hat{A}_{n}^{\dagger}|s\rangle_{n}=\sqrt{(s+1)\left(1-\frac{s}{2 \ell}\right)}|s+1\rangle_{n} .
$$


The spin coherent state $|z\rangle_{n}^{C}$ with label $z \in \mathbb{C}$ is defined by

$$
\left(1+\frac{|z|^{2}}{2 \ell}\right)^{\ell}|z\rangle_{n}^{C}=\exp \left(z \hat{A}_{n}^{\dagger}\right)|0\rangle_{n}=\sum_{s=0}^{2 \ell} \sqrt{\left(\begin{array}{c}
2 \ell \\
s
\end{array}\right)}\left(\frac{z}{\sqrt{2 \ell}}\right)^{s}|s\rangle_{n} .
$$

We also allow a spin coherent state

$$
|\infty\rangle_{n}^{C}=\lim _{z \rightarrow \infty}|z\rangle_{n}^{C}=|2 \ell\rangle_{n}^{C} .
$$

For arbitrary $\zeta \in \mathbb{C}$, the discrete complex-order Fourier transform $\hat{F}_{n}(\zeta)$ is defined to be the operator on $L^{2}(\mathbb{R})$ such that

$$
\hat{F}_{n}(\zeta)|s\rangle_{n}=\zeta^{s}|s\rangle_{n} .
$$

Using Eqs. (30) and (31), followed by Lemma 4.1 and Proposition 4.8, it can be shown that

$$
\hat{F}_{n}(\zeta)=\rho_{n}(K(\zeta)), \quad K(\zeta)=\frac{1}{2}\left(\begin{array}{cc}
1+\zeta & 1-\zeta \\
1-\zeta & 1+\zeta
\end{array}\right)
$$

Evidently, we have a composition law

$$
\hat{F}_{n}(\zeta) \hat{F}_{n}\left(\zeta^{\prime}\right)=\hat{F}_{n}\left(\zeta \zeta^{\prime}\right)
$$

The semigroup $\{\hat{F}(\zeta): \zeta \in \mathbb{C}\}$ is called the discrete Hermite semigroup. Letting $t$ be as in Eq. (61), we say that $\hat{F}_{n}(\zeta)$ has order $t$. The real part of $t$ is still well-defined only up to congruence modulo 1, but there are now no constraints on the range of $t$. By Eq. (50),

$$
\hat{F}_{n}(\zeta)=\exp \left(2 \pi i t \hat{N}_{n}\right)
$$

The discrete fractional Fourier transforms are precisely the unitary discrete complex-order Fourier transforms. By Eqs. (63) and (59), the discrete Hermite semigroup permutes the spin coherent states (up to scalar factors) according to the equation

$$
\hat{F}_{n}(\zeta)|z\rangle_{n}^{C}=\left(\frac{2 \ell+|\zeta z|^{2}}{2 \ell+|z|^{2}}\right)^{\ell}|\zeta z\rangle_{n}^{C}
$$

Theorem 6.1: Given $z \in \mathrm{C}$, then $|z\rangle^{C}=\lim _{n}|z\rangle_{n}^{C}$.

Proof: Consider a vector $\psi \in L^{2}(\mathbb{R})$ and vectors $\psi_{n} \in L_{n}$ such that the set $\left\{\left\|\psi_{n}\right\|: n \in \mathbb{N}\right\}$ is bounded. By Eq. (53) and Ref. 1, Theorem 3.4, $\psi=\lim _{n} \psi_{n}$ if and only if

$$
\langle s \mid \psi\rangle=\lim _{n}\left\langle s \mid \psi_{n}\right\rangle
$$

for all $s \in \mathbb{N}$. These two equivalent conditions hold when $\psi=|z\rangle^{C}$ and $\psi_{n}=|z\rangle_{n}^{C}$ because

$$
\frac{\exp \left(-|z|^{2} / 2\right)}{\sqrt{s !}}=\lim _{\ell \rightarrow \infty}\left(1+\frac{|z|^{1}}{2 \ell}\right)^{-\ell} \sqrt{\left(\begin{array}{c}
2 \ell \\
s
\end{array}\right)}\left(\frac{1}{\sqrt{2 \ell}}\right)^{s} .
$$

Theorem 6.2: Given $\zeta \in \mathrm{C}$ with $|\zeta| \leqslant 1$, then $\hat{F}(\zeta)=\lim _{n} \hat{F}_{n}(\zeta)$.

Proof: Let $\psi \in L^{2}(\mathbb{R})$ and $\psi_{n} \in \mathcal{L}_{n}$ such that $\psi=\lim _{n} \psi_{n}$. Using the criterion for limits noted in the previous argument, 


$$
\langle s \mid \hat{F}(\zeta) \psi\rangle=\zeta^{s}\langle s \mid \psi\rangle=\lim _{n} \zeta^{s}{ }_{n}\left\langle s \mid \psi_{n}\right\rangle=\lim _{n}\left\langle s \mid \hat{F}_{n}(\zeta) \psi_{n}\right\rangle,
$$

and $\hat{F}(\zeta) \psi=\lim _{n} \hat{F}_{n}(\zeta) \psi_{n}$.

\section{CONCLUSIONS}

We have used inductive limits to express the way in which the discrete scenario and the continuum scenario are related to each other. From a procedural point of view (oriented, say, towards implementation of numerical calculations), the relationships between the two scenarios has two significant aspects: approximation and analogy. Not only do the discrete objects serve as approximations to their corresponding continuum objects, but they are also analogs in the sense that the algebraic structures in the discrete scenario mirror the algebraic structures in the continuum scenario. For the purpose of numerical calculation, that feature is important, because it ensures that errors due to inaccuracy of the approximating formulas are not compounded under repeated composition. Our approach provides some rationale for both of those aspects: inductive limits serve as approximations; they also preserve algebraic structures, specifically, they preserve inner products, operator-vector compositions, and operator-operator compositions.

We propose inductive limits as a way of providing theoretical justification for discrete approximations in cases where precise error analysis would be too difficult. As concrete examples become more complicated, intuition may become unreliable, and a precise criterion for the limits may become increasingly useful. Inductive limits of representations, as in Sec. V and Ref. 4, appears to be applicable to various other limits of representations. See, for instance, Refs. 8 and $32-35$. It is to be expected that, through moderately complicated but routine exercises in epsilonics, the limits of operators in those works can be shown to be inductive limits.

However, to plough through such calculations would be to overlook a more interesting line of study. Limits of representations are more subtle than limits of individual operators. The result (Ref. 4, Theorem 9.4) on convergence of structural constants requires, in addition to convergence of individual operators, an analytic convergence hypothesis. The hypothesis is potentially verifiable, in practice, for concrete examples, but some simplifications may be possible; perhaps it suffices to check the uniformity condition in Ref. 4, Sec. 8 only for a spanning set of infinitesmal generators. Thus, at the time of writing, the criterion for inductive limits of representations should be regarded as subject to simplification or modification.

Besides, in order to be of significant practical use, the theory of inductive limits of representations is in need of general theorems. For a limit of representations $\rho=\lim _{n} \rho_{n}$ (Ref. 4. Proposition 9) asserts that, if $\rho$ is faithful, then $\rho_{n}$ is faithful for large $n$. That result is unlikely to be useful in application to concrete examples, since faithfulness is usually obvious to start with. However, the result may point the way forwards: if $\rho$ is irreducible, must $\rho_{n}$ be irreducible for large $n$ ? To prove theorems, of course, it is sometimes necessary to tinker with definitions. So, again, we conclude that the present criterion for inductive limits of representations should be regarded as subject to change.

It appears that inductive limits can also be used to describe a correspondence between a finite-discrete periodic scenario based on the integers modulo $p^{m}$ and a continuous periodic scenario based on the $p$-adic integers. Here, $p$ is a rational prime. For the discrete context, see Refs. 36 and 37; for the continuous context, see Refs. 38, and 39. In this $p$-adic scenario, purely intuitive arguments are to be distrusted, so the use of some or another precise notion of limit is essential.

Discrete versus continuum correspondences of operators and representations arise frequently. Without attempting to classify the various directions of study, let us list some papers on the topic where the term limit is used explicitly and is probably interpretable as inductive limit: Refs. 40, 6, $7,8,32,10,41,42,5,43,44,35$, and $45-47$. We have given a broad spread of citations so as to provide evidence that an intuitive equivalent of the notion of an inductive limit is in widespread use. The list could be extended considerably. The author has come across only one paper (citation 
omitted) in which the limits of operators are nonsensical (arbitrary SL $(2, R)$ canonical transforms written as "limits" of $\operatorname{SL}(2, p)$ canonical transforms, where $p$ runs over the rational primes).

There are also a great many works where the term limit is not used for our purpose, but inductive limits seem to be involved implicitly. This point pertains, in particular, to many singleparameter discrete systems used as approximations to continuum systems in signal processing. For some examples, see Refs. 48 and 49 and references therein.

\section{APPENDIX A: A COUNTER-EXAMPLE}

By the definition of inductive limits of operators, Theorem 6.2 can be expressed as follows.

Theorem A.1: Given a vector $\psi \in L^{2}(\mathbb{R})$ and vectors $\psi_{n} \in \mathcal{L}_{n}$ such that $\psi=\lim _{n} \psi_{n}$, then, for all $\zeta \in \mathrm{C}$ with $|\zeta| \leqslant 1$, we have $\hat{F}(\zeta) \psi=\lim _{n} \hat{F}_{n}(\zeta) \psi_{n}$.

For arbitrary $\zeta \in \mathrm{C}^{\times}$, we can still define $\hat{F}(\zeta)$ to be the operator on $L^{2}(\mathbb{R})$ satisfying Eq. (59). If $|\zeta|>1$, then $\hat{F}(\zeta)$ is unbounded. The domain of $\hat{F}(\zeta)$, in this case, has been studied by Byun. ${ }^{50}$ Plainly, for arbitrary $\zeta$, the conclusion of Theorem A.1 still holds when $\psi$ is a Glauber cat state (linear combination of coherent states) in $L^{2}(\mathbb{R})$ and $\psi$ is the corresponding spin cat state in $\mathcal{L}_{n}$. However, for arbitrary $\zeta$ and arbitrary $\psi$ in the domain of $\hat{F}(\zeta)$, the conclusion of Theorem A.1 can fail. A counter-example is $\psi=0$ and $\psi_{n}=2^{-2 \ell}|2 \ell\rangle$ with $\zeta=3$.

It is difficult to imagine how the mainstream techniques (formal manipulation) could be used to "derive" Theorem A.1 without also "deriving" the fallacy refuted in the previous paragraph.

\section{APPENDIX B: IDENTITIES FOR THE KRAVCHUK FUNCTIONS}

Let $n$ be a positive integer. As in Sec. IV, we write $n=2 \ell+1$ and $[n]=\{-\ell, 1-\ell, \ldots, \ell$ $-1, \ell\}$ and we define $c_{k+1 / 2}=(\ell-k)(\ell+k+1)$, equivalently, $c_{k-1 / 2}=(\ell+k)(\ell-k+1)$, where $2 k \in \mathbb{Z}$. The Kravchuk polynomials $K_{0, n}, K_{1, n}, \ldots, K_{2 \ell, n}$ are the functions $\{0,1, \ldots, 2 \ell\} \rightarrow \mathrm{C}$ given by

$$
\begin{aligned}
K_{\ell+j, n}(\ell+k) & =\left(\begin{array}{c}
2 \ell \\
l+j
\end{array}\right)^{-1} \sum_{\mu=\max (0, j+k)}^{\min (\ell+j, \ell+k)}\left(\begin{array}{c}
\ell+k \\
\mu
\end{array}\right)\left(\begin{array}{c}
\ell-k \\
\ell+j-\mu
\end{array}\right)(-1)^{\mu} \\
& =\sum_{\nu=0}^{\min (\ell+j, \ell+k)}\left(\begin{array}{c}
2 \ell \\
\nu
\end{array}\right)^{-1}\left(\begin{array}{c}
\ell+j \\
\nu
\end{array}\right)\left(\begin{array}{c}
\ell+k \\
\nu
\end{array}\right)(-2)^{\nu},
\end{aligned}
$$

where $j, k \in[n]$. Note that, in each of the two formulas, the index $\mu$ or $\nu$ runs over all values for which the terms are defined. In Sec. IV, it is shown that the two formulas are equivalent to each other. It is also shown that the Kravchuk polynomials satisfy

$$
\begin{gathered}
K_{\ell+k, n}(\ell+j)=K_{\ell+j, n}(\ell+k), \\
K_{\ell+j, n}(\ell-k)=(-1)^{\ell+j} K_{\ell+j, n}(\ell+k), \\
K_{\ell-j, n}(\ell+k)=(-1)^{\ell+k} K_{\ell+j, n}(\ell+k), \\
(\ell-k) K_{\ell+j, n}(\ell+k+1)+2 j K_{\ell+j, n}(\ell+k)+(\ell+k) K_{\ell+j, n}(\ell+k-1)=0, \\
(\ell-j) K_{\ell+j+1, n}(\ell+k)+2 k K_{\ell+j, n}(\ell+k)+(\ell+j) K_{\ell+j-1, n}(\ell+k)=0 .
\end{gathered}
$$

The Kravchuk functions $h_{0, n}, h_{1, n}, \ldots, h_{2 \ell, n}$ are the functions $[n] \rightarrow \mathrm{C}$ given by

$$
h_{s, n}(k)=\frac{(-1)^{s}}{2^{\ell}} \sqrt{\left(\begin{array}{c}
2 \ell \\
s
\end{array}\right)\left(\begin{array}{c}
2 \ell \\
\ell+k
\end{array}\right)} K_{s, n}(\ell+k) .
$$

In other words, 


$$
\begin{aligned}
h_{\ell+j, n}(k) & =\frac{(-1)^{\ell+j}}{2^{\ell}} \sqrt{\frac{(\ell+j) !(\ell-j) !}{(\ell+k) !(\ell-k) !}} \sum_{\mu}\left(\begin{array}{c}
\ell+k \\
\mu
\end{array}\right)\left(\begin{array}{c}
\ell-k \\
\ell+j-\mu
\end{array}\right)(-1)^{\mu} \\
& =\frac{(-1)^{\ell+j}}{2^{\ell}} \sqrt{\frac{(\ell+j) !(\ell+k) !}{(\ell-j) !(\ell+k) !}} \sum_{\nu} \frac{(2 \ell-\nu) !(-2)^{\nu}}{\nu !(\ell+j-\nu) !(\ell+k-\nu) !} .
\end{aligned}
$$

Equations (B1-B5) can be rewritten as

$$
\begin{gathered}
h_{\ell+k, n}(j)=(-1)^{k-j} h_{\ell+j, n}(k), \\
h_{\ell+j, n}(-k)=(-1)^{\ell+j} h_{\ell+j, n}(k), \\
h_{\ell-j, n}(k)=(-1)^{\ell-k} h_{\ell+j, n}(k), \\
\sqrt{c_{k+1 / 2}} h_{\ell+j, n}(k+1)+2 j h_{\ell+j, n}(k)+\sqrt{c_{k-1 / 2}} h_{\ell+j, n}(k-1)=0, \\
\sqrt{c_{j+1 / 2}} h_{\ell+j+1, n}(k)-2 k h_{\ell+j, n}(k)+\sqrt{c_{j-1 / 2}} h_{\ell+j-1, n}(k)=0 .
\end{gathered}
$$

Proposition 4.7 says that Kravchuk functions comprise an orthonormal basis for the space of functions $[n] \rightarrow \mathrm{C}$.

${ }^{1}$ L. Barker, J. Funct. Anal. 186, 153 (2001).

${ }^{2}$ L. Barker, J. Phys. A 22, 4673 (2001).

${ }^{3}$ L. Barker, J. Math. Phys. 42, 4653 (2001).

${ }^{4} \mathrm{~L}$. Barker, Limits of representations of Lie groups and Lie algebras (submitted).

${ }^{5}$ J. M. Radcliffe, J. Phys. A 4, 313 (1971).

${ }^{6}$ F. T. Arecchi, E. Courtans, R. Gilmore, and H. Thomas, Phys. Rev. A 6, 2211 (1972).

${ }^{7}$ N. Atakishiyev, S. M. Chumakov, and K. B. Wolf, J. Math. Phys. 39, 6247 (1998).

${ }^{8}$ N. Atakishiyev, G. S. Pogosyan, L. E. Vicent, and K. B. Wolf, J. Phys. A 34, 9381 (2001).

${ }^{9}$ V. K. Dobrev, H.-D. Doebner, and C. Mrugalla, Mod. Phys. Lett. A 14, 1113 (1999).

${ }^{10}$ N. M. Atakishiyev and K. B. Wolf, J. Opt. Soc. Am. A 14, 1467 (1997).

${ }^{11}$ T. Digernes, V. S. Varadarájan, and S. R. S. Varadhan, Rev. Math. Phys. 6, 612 (1994).

${ }^{12}$ P. Kramer and M. Lorente, "Discrete and continuous symmetry via induction and duality," in Symmetries in Science, $X$ (Bregenz, 1997) (Plenum, New York, 1998), pp. 165-177.

${ }^{13}$ K. R. Parthasarathy, J. Appl. Probab. Special Vol. 25A, 151 (1988).

${ }^{14}$ K. R. Parthasarathy, An Introduction to Quantum Stochastic Calculus (Birkhäuser, Basel, 1992).

${ }^{15}$ J. M. Lindsay and K. R. Parthasarathy, Sankhya, Ser. A 50, 151 (1988).

${ }^{16}$ U. Neiderer, Helv. Phys. Acta 45, 802 (1972).

${ }^{17}$ V. Namias, J. Inst. Math. Appl. 25, 214 (1980).

${ }^{18}$ G. B. Folland, Harmonic Analysis in Phase Space (Princeton University Press, Princeton, NJ, 1989).

${ }^{19}$ M. Hillery, R. F. O'Connell, M. O. Scully, and E. P. Wigner, Phys. Rep. 106, 121 (1984).

${ }^{20}$ R. G. Littlejohn, Phys. Rep. 138, 193 (1986).

${ }^{21}$ A. M. Ozorio de Almeida, Phys. Rep. 295, 265 (1998).

${ }^{22}$ G. W. Forbes, V. I. Man'ko, H. M. Ozaktas, R. Simon, and K. B. Wolf (eds.), "Wigner Distributions and Phase Space in Optics," J. Opt. Soc. Am. A (feature issue) 17(12), 2274 (2000).

${ }^{23}$ The Wigner Distribution, Theory and Applications in Signal-Processing, edited by W. Mecklenbräuker and F. Hlawatsch (Elsevier, Amsterdam, 1996).

${ }^{24}$ N. Ja. Vilenkin and A. U. Klimyk, Representation of Lie Groups and Special Functions, Vol. 1 (Kluwer, Dordrecht, 1993).

${ }^{25}$ A. Wawrzyńczyk, Group Representations and Special Functions (Kluwer, Dordrecht, 1978, 1984).

${ }^{26}$ M. Lorente, Phys. Lett. A 285, 119 (2001).

${ }^{27}$ M. Lorente, El. Trans. Numer. Anal. 9, 102 (1999).

${ }^{28}$ J. R. Klauder and B-S. Skagerstam, Coherent States, Applications in Physics and Mathematical Physics (World Scientific, Singapore, 1985).

${ }^{29}$ W-M. Zhang, D. H. Feng, and R. Gilmore, Rev. Mod. Phys. 62, 867 (1990).

${ }^{30}$ F. B. Weissler, J. Funct. Anal. 32, 102 (1979).

${ }^{31}$ C-C. Shih, Opt. Lett. 20, 1178 (1995).

${ }^{32}$ N. Atakishiyev, G. S. Pogosyan, L. E. Vicent, and K. B. Wolf, J. Phys. A 34, 9399 (2001).

${ }^{33}$ M. Lorente and P. Kramer, "Induced representations of the Poincaré group on the lattice: spin 1/2 and spin 1 case," in Symmetries in Science. X (Bregenz, 1997) (Plenum, New York, 1998), pp. 179-195.

${ }^{34}$ M. Lorente and P. Kramer, J. Phys. A 32, 2481 (1999). 
${ }^{35}$ M. Ruzzi and D. Galetti, J. Phys. A 33, 1065 (2000).

${ }^{36}$ M. S. Richman and T. W. Parks, "Understanding discrete rotations," in Proc. 1997 IEEE Int. Conf. Acoustics, Speech and Signal Processing (IEEE, Piscataway, NJ 1997).

${ }^{37}$ M. S. Richman, T. W. Parks, and R. G. Shenoy, IEEE Trans. Signal Process. 46, 1517 (1998).

${ }^{38}$ A. Bechata, C. R. Acad. Sci., Ser. I: Math. 326, 157 (1998).

${ }^{39}$ V. S. Vladimirov, I. V. Volovich, and E. I. Zelenov, p-Adic Analysis and Mathematical Physics (World Scientific, Singapore, 1994)

${ }^{40}$ R. Aldrovandi and D. Galetti, J. Math. Phys. 31, 2978 (1990).

${ }^{41}$ D. B. Fairlie and C. K. Zachos, Phys. Lett. B 224, 101 (1989).

${ }^{42}$ D. Galetti and A. F. R. de Toledo Piza, Physica A 214, 207 (1995).

${ }^{43}$ A. M. F. Rivas and A. M. Ozorio de Almeida, Ann. Phys. 276, 223 (1999).

${ }^{44}$ M. Ruzzi, J. Phys. A 35, 1763 (2002).

${ }^{45}$ M. Ruzzi and D. Galetti, J. Phys. A 35, 4633 (2002).

${ }^{46}$ J. A. Vaccaro and D. T. Pegg, Phys. Rev. A 41, 5156 (1990).

${ }^{47}$ V. R. Vieira and P. D. Sacramento, Ann. Phys. 242, 188 (1995).

${ }^{48}$ S-C. Pei and M-H. Yeh, IEEE Trans. Signal Process. 49, 1198 (2001).

${ }^{49}$ C. Richard, IEEE Trans. Signal Process. 49, 2536 (2001).

${ }^{50}$ D-W. Byun, Proc. Am. Math. Soc. 118, 437 (1993). 